$\mathcal{G S}_{\text {https://doi.org/10.3765/sp.11.4 }}^{\text {Semantics \& Pragmatics Volume 11, Article 4, } 2018}$

This is an EARLY ACCESS version of

Nouwen, Rick. 2018. Free choice and distribution over disjunction: the case of free choice ability. Semantics and Pragmatics 11(4). https://doi.org/10. 3765/sp.11.4.

This version will be replaced with the final typeset version in due course. Note that page numbers will change, so cite with caution. 


\title{
Free choice and distribution over disjunction: the case of free choice ability*
}

\author{
Rick Nouwen \\ Utrecht University
}

\begin{abstract}
This squib discusses the semantics of ability modals in relation to the law of distribution over disjunction and free choice effects. Most current analyses of free choice need distribution over disjunction as a theorem for modals in order to correctly derive free choice inferences. Famously, however, ability modals have been argued to fail to meet distribution over disjunction (Kenny 1976). The squib explores to what extent free choice abilities are dependent on the distribution property.
\end{abstract}

Keywords: free choice, disjunction, ability, homogeneity

\section{Free choice disjunction}

The classical puzzle of free choice permission is that while (1) is not valid in any classical deontic logic, it seems valid for the corresponding natural language sentences, as in (2) (Kamp 1973).

$$
\diamond[p \vee q] \Rightarrow \diamond p \wedge \diamond q
$$

(2) You may pick an apple or a pear

a. $\Rightarrow$ You may pick an apple

b. $\Rightarrow$ You may pick a pear.

The effect turns out to be independent of modal flavour (for instance, Zimmermann 2000) and, in fact, has even been argued to be independent from modality (for instance, Klinedinst 2007, Fox 2007). The generality of the phenomenon is one reason why large parts of the literature seek an explanation of the free choice effect in the interaction of existential quantificational force and disjunction. In this squib, I explore the generality of the phenomenon by turning to a particular constraint on free choice inferences that is left implicit in a dominant approach.

* The research leading to these results has received funding from the European Research Council under the European Union's Seventh Framework Programme (FP/2007-2013) / ERC Grant Agreement no. 313502, which I hereby gratefully acknowledge. For discussion and comments, I would like to thank Bart Geurts, John Horty, Nathan Klinedinst, Matthew Mandelkern, Daniel Rothschild, the editor in charge, Paul Elbourne and the anonymous reviewers. 


\section{Distribution over disjunction}

There are (roughly) two kinds of approaches to free choice. On the one hand, there are approaches that think the free choice effect is due to lexical semantics, either of disjunction (Zimmermann 2000, Geurts 2005) or of the existential operator (Aloni 2003, Simons 2005, Barker 2011). I will have nothing to say about these approaches. Instead, this squib concerns the large strand of literature that treats free choice inferences as an inference resulting from reasoning about alternatives.

Reasoning about alternatives is one of the central topics of Semantics and Pragmatics. There are profound disagreements about what exactly such reasoning involves and which level of interpretation it is part of (for instance, Chierchia et al. 2011, Geurts 2009, Geurts \& Pouscoulous 2009, Geurts 2011, Chemla \& Spector 2011, Franke 2009, Frank \& Goodman 2012). Given these disputes, it is remarkable that there is a peaceful consensus about the mechanism involved in free choice. Despite the vast array of differences in approaches to alternatives and inferences based on them, the core idea of how free choice should be accounted for is the same irrespective of which corner of the field it is implemented in.

The source of this idea can be traced back to Kratzer \& Shimoyama (2002). It rests on the following minimal assumptions, put in theory-neutral terms: (i) there are meanings that come about via reasoning about alternatives; (ii) The individual disjuncts of a disjunction $p \vee q$ are themselves alternatives to that disjunction; and (iii) this type of reasoning can be iterative.

Take a proposition like (3) as the conventional meaning of a speaker's utterance:

$$
\diamond[p \vee q]
$$

A hearer may now reason about the speaker's intentions: why did the speaker use a disjunction and not simply one of the disjuncts (alternatives)? She then concludes that the speaker didn't convey the simpler message $\diamond p$ (individual disjunct) for one of two reasons: either $\diamond p$ was false or - and this is the iterative aspect $-\diamond p$ would have lead to the false conclusion by the hearer that $\diamond p$ was the only permission to be had. In particular, $\diamond p$ may trigger the reasoning that the alternative $\diamond q$ is false. So, there are now two possible reasons why $\diamond p$ was not conveyed: either it is false, or the inference we would draw from it, $\neg \diamond q$, is false. In other words, the hearer concludes that $\neg \diamond p \vee \diamond q$. She does the same for the other disjunct yielding $\neg \diamond q \vee \diamond p$. Together, we now have the implicature in (4):

$$
\diamond p \leftrightarrow \diamond q
$$

If we left things here, we'd predict a pretty strange reading for free choice statements: either both disjuncts are permitted or both are not permitted: 
Free choice and distribution over disjunction

$$
(\diamond p \wedge \diamond q) \vee(\neg \diamond p \wedge \neg \diamond q)
$$

However, since the assertion in (3) says that at least one of the disjuncts is permitted, it follows from (5) that both are permitted. And, so, the free choice inference is accounted for.

This final crucial step is what I want to focus on in the following. I assumed here that (3) entails that either $p$ or $q$ is permitted. This is known as distribution over disjunction, which I will abbreviate as DOD:

$$
\diamond[p \vee q] \Rightarrow \diamond p \vee \diamond q
$$

DOD

In standard modal logics, distribution over disjunction is a theorem. For instance, in a possible world semantics for modals, (6) follows from its semantics: if there is no accessible $p$-world and no accessible $q$-world, then there cannot be an accessible $p \vee q$-world. While the Kratzer-Shimoyama style reasoning leads to (5), it is only through the DOD theorem in (6) that the combination of (3) and (5) is strengthened to the free choice inference $\diamond p \wedge \diamond q$. $^{1}$

As I said, Kratzer and Shimoyama's idea has been implemented in strongly opposing theoretical corners. ${ }^{2}$ To illustrate, let me go through what the same mechanism looks like in a quite different setup. The account of free choice implicatures in Geurts (2011) is framed differently, but follows a reasoning pattern that is quite similar to that of Kratzer \& Shimoyama (2002). The idea is that hearers reason about the intentional state of the speaker. For a sentence $\diamond[p \vee q]$ the hearer may entertain one of four possibilities: $\left(i_{1}\right) \diamond p \wedge \diamond q,\left(i_{2}\right) \diamond p \wedge \neg \diamond q,\left(i_{3}\right) \diamond q \wedge \neg \diamond p,\left(i_{4}\right)$ $\neg \diamond p \wedge \neg \diamond q$. That is, here the alternatives are not alternative statements, but rather alternative (classes of) states of affairs. These different states vary with respect to whether or not the individual disjuncts are possibilities. To be clear: these intentional possibilities have an altogether different status in Geurts' theory than alternative utterances or grammatical structures in our discussion above, (which are the kind of alternatives that, traditionally, receives this label in the literature). Yet, from a purely logical point of view, they play a similar role in getting to the end-result of a free choice inference.

Given DOD, the hearer may dismiss $i_{4}$ straight away since it is not compatible with the speaker's assertion that $\diamond[p \vee q]$ is true. She then reasons that $i_{2}$ and $i_{3}$ can also be disregarded, because if the speaker had been in one of these states she would

1 The above description of the mechanism is quite closely implemented in the prominent and widely used theory of Fox (2007), who implements the free choice recipe in a grammatical mechanism for implicature. It straightforwardly inherits the prediction that free choice would not arise without DOD. For the initiated, technically, without DOD the alternatives $\diamond p$ and $\diamond q$ are innocently excludable, leading to the implicature $\neg \diamond p \wedge \neg \diamond q \wedge \neg \diamond[p \wedge q]$, quite the opposite of free choice.

2 For this reason, I am tempted to call this status quo the pax Kratzer-Shimoyama. 
have said something shorter, namely something that simply conveyed $\diamond p$ or $\diamond q$, respectively. This way, the hearer arrives at the conclusion that the speaker must be in state $i_{1}$, the state where the free choice inference holds. This result crucially relies on the hearer being able to dismiss $i_{4}$. If DOD does not hold, only $i_{2}$ and $i_{3}$ can be excluded, thus leading to the implicature (5). Quite a similar issue arises in Franke (2009, 2011) and van Rooij (2010), since these are also frameworks that rely on dividing up a logical space. Were DOD invalid, it would make the modal statement consistent with more partition cells than if DOD had been valid.

DOD is tacitly assumed for modals in the free choice literature. Because it is a theorem of the standard semantics for existential modals, there is simply no discussion. If the approaches that follow the Kratzer-Shimoyama reasoning are on the right track, then we would expect the occurrence of free choice inferences to rely on whether or not distribution over disjunction holds.

How do we test this prediction? One route would be to look at operators intervening between the existential (modal) quantifier and disjunction that disrupt the validity of DOD. While DOD holds for $\diamond[p \vee q]$ it does not hold for $\diamond \square[p \vee q]$ :

$$
\diamond \square[p \vee q] \nRightarrow \diamond \square p \vee \diamond \square q
$$

Just imagine a world in which only one other world is accessible, while from that latter world we may access a $p \wedge \neg q$ world and a $q \wedge \neg p$ world. This makes $\diamond \square[p \vee q]$ true, but neither $\diamond \square p$ nor $\diamond \square q$.

Unfortunately, we cannot test directly whether an operator with universal force intervening between existential quantification and disjunction blocks free choice. This is because we know that disjunction readily takes scope wider than surface scope. In fact, it seems immune to any constraint on scope taking. If we did see free choice for a sentence of the surface form $\diamond>\square>\vee$ it could simply be that it was interpreted as $\diamond>\vee>\square$, for which free choice follows straightforwardly. In order to test the DOD-dependency of free choice we therefore need to turn to lexical combinations of existential and universal force.

\section{Ability and distribution over disjunction}

As Kenny (1976) famously argued, distribution over disjunction is invalid for ability modals. The argument goes as follows. Say there is a deck of regular playing cards in front if you. The cards are face down, concealing the colours. If you were to pick one of these cards, it would either be red or black. You obviously have the ability to pick a card, so (8) is true. However, if DOD holds, it should follow from that that either (9) or (10) is true. But unless you are a skilled conjuror, you simply won't have the ability to pick a card and predict its color. Whether the card you pick is red or black relies completely on chance. 
Free choice and distribution over disjunction

(8) I can bring it about that either I am picking a red card or I am picking a black card.

(Kenny 1976, p. 215)

(9) I can bring it about that I pick a black card.

(10) I can bring it about that I pick a red card.

Kenny's argument targets analyses that characterise the concept of ability in terms of simple quantification over worlds: Such analyses wrongly predict distribution over disjunction to hold. Given my current focus on free choice inferences, however, we can take the argument one step further. Given the fact that Kenny observes that the disjunction of (9) and (10) does not follow from (8), their conjunction does not follow either. Kenny's example (8), in other words, shows an absence of ability free choice in tandem with an absence of distribution over disjunction. As we will see below, Kenny's observations regarding (8) are not representative of all ability statements. ${ }^{3}$ For now, however, I would like to focus on the how the theoretical developments triggered by Kenny's observations tie in with the recent free choice literature.

In part due to Kenny's observations there have been many proposals that give a semantics of ability that does not yield distribution over disjunction (for instance, among many others Horty \& Belnap 1995, Thomason 2005.) The intuition behind many of these is the following: someone is able to $p$ means that there is some action that will reliably result in $p$ being true. ${ }^{4}$ With such a view of ability, DOD no longer holds. If some action reliably makes $p \vee q$ true, this does not entail that there is an action that reliably brings $p$ about or that there is an action that reliably brings $q$ about. For instance, Brown (1988) uses a semantics that has models made up of a set of worlds $W$ and a function $N$ that maps worlds to propositions (the available actions in that world). Ability is now interpreted as follows:

$\alpha$ has the ability to $A$ in $w$ if and only if $\exists m \in N(w): \forall w \in m: A(w)$.

Portner (2009) suggests that within a Kratzerian framework there is the option of assigning ability modals like can the semantics of good possibility, which has this same structure of a combination of existential and universal force.

3 As an anonymous reviewer rightly observes, for instance, that intuitions for a minimal variation on (8) are not so clear: "I can pick a red or a black card from the stack" is pretty odd when the speaker does not have the ability of choosing which colour he picks (i.e. when she's not a conjuror).

4 As two reviewers rightly point out, such a view only works when the available actions are somehow linked to the agent. That is, the actions in question have to be actions controlled by the agent. Because what is important to the present paper is mainly the quantificational structure of ability modals, I gloss over such important details. 
(12) For a modal base $F$ and an ordering source $g: p$ is a good possibility w.r.t. $\langle F, g\rangle$ if and only if $\exists w \in F \forall w^{\prime}\left[w>_{g} w^{\prime} \rightarrow p\left(w^{\prime}\right)\right]$.

Clearly, $p \vee q$ can be a good possibility even if neither $p$ nor $q$ is a good possibility. What good possibility shares with Brown's action semantics is that it combines existential and universal force. Assuming ability indeed involves such a combination, then DOD is invalid. So the prediction is that there will be no free choice ability. Unfortunately, however, and in contrast to Kenny's observation for (8), this prediction is not borne out, as is demonstrated by (13), from Geurts (2011).

Betty can balance a fishing rod on her nose or on her chin.

Without a doubt, (13) has a free choice inference: We easily infer from (13) that Betty is capable of balancing a fishing rod on her chin, for instance. We now have an argument that Kratzer/Shimoyama-style approaches to free choice make the problematic prediction that there cannot be free choice ability. As I will show in the remainder of this squib, there are several subtleties muddling this argument.

\section{Homogeneity and accidental success}

Kenny's argument against the validity of DOD for ability modals was based on the fact that someone's accidental success at bringing something about does not give that person the ability to bring this about. For some abilities, however, the idea of accidentally succeeding does not really make sense. As I will show, this in turn means that for these abilities DOD $i$ v valid. The best way to see this is to consider negated ability statements. Compare (14) and (15). 5,6

(14) John can't balance a fishing rod on his nose.

$$
\text { John can't hit the bull's eye. }
$$

We have assumed that can involves some combination of existential and universal force. Let's say that $\boldsymbol{\square}$ [A fishing rod is balancing on John's nose] stands for there is a way for John to act that reliably makes the fishing rod balance on his nose. Such an account would say that (14) means that there is no way that reliably makes the fishing rod stand upright on John's nose. This leaves open the option that some actions may result in a balancing fishing rod, just not reliably so. However, we know

$5 \mathrm{I}$ am indebted to an anonymous reviewer for urging me to rethink the discussion below.

6 The discussion below is inspired by Nickel (2010), who develops an analysis of free choice effects for generic statements. Nickel's semantics for such statements also combines existential and universal force. Although he does not discuss DOD, he does observe that his proposal predicts there to be no free choice inferences. His solution is in some ways parallel to what I will now discuss for ability modals. 
that if you lack the ability to balance the rod, gravity will make sure that the rod will fall down. Fishing rods don't accidentally stay upright. This is another way of saying that balancing-abilities are homogeneous: for any action, if it is the right one it will reliably result in a balancing rod; if it is not, it will reliably result in the rod falling down.

Things are different for (15), however. Imagine this sentence is about John's abilities of playing darts. Imagine that (15) is true and that John lacks the ability to precisely aim a dart at a darts board. Then he may still hit the bull's eye by accident if he tried. This means that this kind of ability is not homogeneous. The fact that he may hit the bull's eye is inconsequential to his ability. The only thing that matters is that he cannot reliably hit the bull's eye. He therefore lacks the relevant ability.

In sum, homogeneity for ability is tied with the absence or presence of accidental success: it holds for some cases, but definitely not for all. When homogeneity does hold, it looks like (16). This will allow us to strengthen (17a) to (17b), and thus we end up concluding from (14) that any action will reliably cause the rod to fall down.

$$
\begin{aligned}
& \mathbf{\square}[\boldsymbol{\square} \vee \square \neg \varphi] \\
& \text { a. } \neg \nabla \mathbf{\square} \\
& \text { b. } \square \neg p
\end{aligned}
$$$$
\text { homogeneous ability }
$$

Homogeneity also predicts the presence of free choice inferences. ${ }^{7}$ According to the Kratzer-Shimoyama recipe we get the inference in (18) for an ability statement interpreted as $\bullet \mathbf{v}[p \vee q]$.

$$
(\diamond \mathbf{m} p \wedge \triangleright \mathbf{m} q) \vee(\neg \diamond \mathbf{m} p \wedge \neg \diamond \mathbf{m} q)
$$

According to (16) this will be strengthened to:

$$
(\diamond \mathbf{\square} p \wedge \diamond \mathbf{\square}) \vee(\mathbf{\square} \neg p \wedge \mathbf{\square} \neg q)
$$

Clearly the second disjunct is incompatible with the statement that $\bullet \mathbf{\square}[p \vee q]$ since both $p$ and $q$ are reliably false after any action, and, consequently, free choice follows. This result will not come as a surprise as soon as we realise that the assumption of homogeneity, as in (16), makes DOD valid. Considering $p$ and $q$, homogeneity says that $\boldsymbol{\square}[\boldsymbol{\square} p \vee \boldsymbol{\square} \neg p]$ and $\boldsymbol{\square}[\boldsymbol{\square} q \vee \boldsymbol{\square} \neg q]$. If we now consider cases in which $\bullet \boldsymbol{\square}[p \vee q]$ is true, we see that, from some accessible world, $p \vee q$ can only be true in every subsequently accessible world if $p$ is true in all these worlds or $q$ is true in all these worlds (or both). A situation in which $\bullet \mathbf{\square}[p \vee q]$ is true, but neither $\bullet \mathbf{q} p$ nor $\bullet \mathbf{q} q$ is true is simply ruled out, due to homogeneity.

7 Again, see Nickel (2010) for a parallel argument for generic statements. 
Taking stock, we now know the following: (i) According to the Kratzer \& Shimoyama-inspired approach to free choice, the validity of DOD is a prerequisite of free choice. (ii) If we follow the analyses that attribute the quantificational force of $\checkmark$ to abilities, then, logically abilities do not make DOD valid. (iii) Some abilities are homogeneous, however, and through homogeneity, they do make DOD valid. On the other hand, for other abilities, as in (15), homogeneity cannot be assumed.

Given this, I can envision two possible theories based on these assumptions. First, if we simply take these points at face value, then we come to predict that free choice occurs in all and only those ability statements that involve abilities without accidental success. A second possible take could be that homogeneity is somehow instantiated whenever ability ascriptions are made. ${ }^{8}$ That is, accidental success is excluded whenever an ability statement is made. If this were the case, then distribution over disjunction would hold for all such statements, and so free choice would be predicted to arise generally, just like it arises generally for deontic and epistemic statements.

I think we can dismiss the second of these theories quite easily, for two reasons. First of all, the intuition for (15) is that it is true even if John might accidentally hit the bull's eye. So we cannot assume homogeneity to hold in this case. Second, there are clear cases where free choice ability does not occur. Kenny's intuitions about (8) are a case in point if you agree with his judgments, but there are more straightforward examples, too. Take (20), which is a decidedly odd way of communicating to the hearer that my son Oscar is now of an age at which he can walk and can talk.

Oscar can walk or talk.

Similarly, there are variations on Geurts' fishing rod example that lose the free choice inference: ${ }^{9}$

(21) Betty can balance a fishing rod on her nose or juggle four hot potatoes with just her left hand.

*FC

Note that (20) and (21) also dismiss the first theoretical line I suggested, which held that free choice is present whenever accidental success is absent. Clearly, both (20) and (21) involve homogeneous abilities, without triggering free choice.

8 Thanks to an anonymous reviewer for urging me to spell this out somewhat.

9 The relative unavailability of free choice inferences for ability statements is sometimes masked by the polysemy of can, i.e. by its ambiguity with respect to modal flavour. If you are told that "John can speak English or German", there is a clear free choice inference on a teleological reading of can. I do not think free choice is available on a ability reading of can. Similarly for (21), I think the example is fine with a free choice inference after a (teleological) question under discussion like "How can Betty entertain the guests?". 
In summary, then, the predicted DOD-dependence of free choice ability is not attested. Instead, what can be observed is that free choice ability is quite a limited phenomenon, but that the limitation seems disconnected from homogeneity / the validity of DOD.

I think what is crucial to good examples of ability free choice is that they typically involve two variations of the same ability, as in Geurts' original example, repeated here as (22).

Betty can balance a fishing rod on her nose or on her chin.

In contrast, (21) contains two distinct abilities, which somehow prohibits free choice. Speculating somewhat, free choice ability always seems to involves a conjunction of inferences that concern the same general ability. This is obviously not a very precise statement and I have no explanation for this observation, but I do have this to say: Whatever accounts for the differences between the example with and those without free choice inferences, it is not the absence versus presence of DOD.

\section{Conclusion}

I have identified two challenges for the semantics and pragmatics of free choice disjunction. First, the dominant Kratzer and Shimoyama-style approaches to how free choice inferences come about need to explain how it is possible ability can at times triggers such inferences without distribution over disjunction being a valid theorem. Secondly, there is an empirical challenge. Ability free choice is a limited phenomenon. This is in stark contrast to other model free choice phenomena (though see Eckardt 2007, Portner 2010). Right now I see no way of making sense of the constraints that appear to govern it.

A shared idea within the approaches I targeted in this squib is that free choice is triggered simply by the logical interplay of existential quantificational force and disjunction. This is obviously an attractive idea, given that it reduces free choice to a very general pragmatic (or, alternatively, grammatical) process. What I hope to have shown is that the fringes of the landscape of existential operators is a challenging testing ground for this way of thinking. At first glance, the dominant approaches fail the test.

\section{References}

Aloni, Maria. 2003. Free choice in modal contexts. In Matthias Weisgerber (ed.), Sinn und Bedeutung ( $\mathrm{SuB}$ ) 7, vol. 114, Konstanz: Universität Konstanz.

Barker, Chris. 2011. Free choice permission as resource-sensitive reasoning. Semantics and Pragmatics 3(1). 1-38. https://doi.org/10.3765/sp.3.10. 
Brown, Mark A. 1988. On the logic of ability. Journal of philosophical logic 17(1). 1-26. http://www.jstor.org/stable/30226385.

Chemla, Emmanuel \& Benjamin Spector. 2011. Experimental evidence for embedded scalar implicatures. Journal of semantics 359-400. https://doi.org/10.1093/ jos/ffq023.

Chierchia, Gennaro, Danny Fox \& Benjamin Spector. 2011. The grammatical view of scalar implicatures and the relationship between semantics and pragmatics. In Paul Portner, Claudia Maienborn \& Klaus von Heusinger (eds.), An international handbook of natural language meaning, Mouton de Gruyter.

Eckardt, Regine. 2007. Licensing or. In Uli Sauerland \& Penka Stateva (eds.), Presupposition and implicature in compositional semantics, 34-70. Palgrave MacMillan. https://doi.org/10.1057/9780230210752_3.

Fox, Danny. 2007. Free choice and the theory of scalar implicatures. In Uli Sauerland \& Penka Stateva (eds.), Presupposition and implicature in compositional semantics, 71-120. Palgrave MacMillan. https://doi.org/10.1057/9780230210752_4.

Frank, Michael C \& Noah D Goodman. 2012. Predicting pragmatic reasoning in language games. Science 336(6084). 998-998. https://doi.org/10.1126/science. 1218633.

Franke, Michael. 2009. Signal to act: game theory in pragmatics: Universiteit van Amsterdam dissertation.

Franke, Michael. 2011. Quantity implicatures, exhaustive interpretation, and rational conversation. Semantics and Pragmatics 4. 1-82. https://doi.org/10.3765/sp.4.1. Geurts, Bart. 2005. Entertaining alternatives: disjunctions as modals. Natural Language Semantics 13. 383-410. https://doi.org/10.1007/s11050-005-2052-4.

Geurts, Bart. 2009. Scalar implicature and local pragmatics. Mind E Language 24(1). 51-79. https://doi.org/10.1111/j.1468-0017.2008.01353.x.

Geurts, Bart. 2011. Quantity implicatures. Cambridge University Press.

Geurts, Bart \& Nausicaa Pouscoulous. 2009. Embedded implicatures?!? Semantics and Pragmatics 2(4). 1-34. https://doi.org/10.3765/sp.2.4.

Horty, John F \& Nuel Belnap. 1995. The deliberative stit: A study of action, omission, ability, and obligation. Journal of philosophical logic 24(6). 583-644. http://www.jstor.org/stable/30227231.

Kamp, Hans. 1973. Free choice permission. Proceedings of the Aristotelian Society 74. 57-74. http://www.jstor.org/stable/4544849.

Kenny, Anthony. 1976. Human abilities and dynamic modalities. In Essays on explanation and understanding, 209-232. Springer. https://doi.org/10.1007/97894-010-1823-4_11.

Klinedinst, Nathan. 2007. Plurality and possibility: University of California Los Angeles dissertation. 
Free choice and distribution over disjunction

Kratzer, Angelika \& Junko Shimoyama. 2002. Indeterminate pronouns: The view from Japanese. In Y. Otsu (ed.), Third Tokyo Conference on Psycholinguistics (TCP), 1-25. Tokyo: Hituzi Syobo.

Nickel, Bernhard. 2010. Generically free choice. Linguistics and philosophy 33(6). 479-512. https://doi.org/10.1007/s10988-011-9087-4.

Portner, Paul. 2009. Modality. OUP Oxford.

Portner, Paul. 2010. Permission and choice. Discourse and grammar. From sentence types to lexical categories 43-68.

van Rooij, Robert. 2010. Conjunctive interpretation of disjunction. Semantics and Pragmatics 3(11). 1-28. https://doi.org/10.3765/sp.3.11.

Simons, Mandy. 2005. Dividing things up: The semantics of or and the modal/or interaction. Natural Language Semantics 13(3). 271-316. https://doi.org/10. 1007/s11050-004-2900-7.

Thomason, Richmond. 2005. Ability, action and context. Ms. University of Michigan.

Zimmermann, Thomas Ede. 2000. Free choice disjunction and epistemic possibility. Natural Language Semantics 8. 255-290. https://doi.org/10.1023/A: 1011255819284.

Rick Nouwen

Trans 10

NL-3512JK, Utrecht, the Netherlands

R.W.F.Nouwen@uu.nl 